\title{
Acute pancreatitis in pregnancy: A 10-year, multi-center, retrospective study in Beijing
}

\section{Tingting Zhang}

Capital Medical University

\section{Guoxing Wang}

Capital Medical University

\section{Zheng Cao}

Capital Medical University

\section{Wenyang Huang}

Capital Medical University

Hongli Xiao

Capital Medical University

Hongtao Wei

Capital Medical University

Junli Lu

Capital Medical University

\section{Ruixia Liu}

Capital Medical University

Chenghong Yin ( $\square$ yinchh@ccmu.edu.cn )

Capital Medical University

\section{Research Article}

Keywords: Acute pancreatitis in pregnancy, Etiology, Clinical manifestations, Maternal outcomes, Fetal outcomes

Posted Date: November 15th, 2021

DOI: https://doi.org/10.21203/rs.3.rs-963386/v1

License: (c) (1) This work is licensed under a Creative Commons Attribution 4.0 International License. Read Full License 


\section{Abstract \\ Objective}

Acute pancreatitis in pregnancy (APIP) is a rare and serious complication during pregnancy. It has acute onset and is difficult to diagnose and treat. The aim of the present study was to describe the etiology, clinical manifestations, and maternofetal outcomes of APIP.

\section{Methods}

We retrospectively reviewed 32 pregnant women who were treated at three tertiary care hospitals in Beijing, China. The correlation between the causes of APIP, severity, laboratory indices, and outcomes was analyzed.

\section{Results}

The most common causes of APIP were hypertriglyceridemia $(56.2 \%, 18 / 32)$ and gallstones $(28.1 \%, 9 / 32)$. Hypertriglyceridemia-induced APIP was associated with a higher rate of severe acute pancreatitis $(P=0.025)$. Serum level of triglycerides showed a positive correlation with the severity of APIP $(P=0.039)$. The most frequent presentation of APIP was abdominal pain (93.7\%, 30/32). There were no maternal or fetal deaths in our study. Neonatal asphyxia was correlated with the severity of APIP $(P=0.039)$.

\section{Conclusion}

High level of triglycerides may serve as a useful marker of the severity of APIP. The severity of APIP was associated with higher risk of neonate asphyxia. Appropriate timing of termination of pregnancy is a key imperative for APIP patients.

\section{Introduction}

Acute pancreatitis in pregnancy (APIP) is one of the rare and serious complications during pregnancy ${ }^{[1-3]}$. The reported incidence rate of APIP varies between 1/12000-1/1000 ${ }^{[1-3]}$. An increase in the incidence rate of APIP has been observed over the past decades ${ }^{[4-7]}$. Acute progression of APIP may lead to pancreatic necrosis, abscess, multi-organ dysfunction, and other adverse maternal and fetal outcomes. APIP has an acute onset and is typically difficult to diagnose and treat. According to a study, APIP may be more harmful to the fetus compared to the mother ${ }^{[7]}$. However, owing to its rarity, most published research on this subject is based on small case series. In addition, most retrospective studies had a relatively long reference time-period; therefore, these studies could not characterize the changes in APIP characteristics in recent years. Furthermore, the features of APIP may vary greatly in different geographical areas and ethnic groups ${ }^{[1,2,7]}$. Of late, there have been great changes in the diagnosis and treatment of APIP; however, there is a paucity of contemporary reports from China on the clinical features of APIP and the outcomes.

In this study, we retrospectively reviewed 32 cases of APIP treated at the following three tertiary care centers in Beijing, China: Beijing Obstetrics and Gynecology Hospital; Beijing Chaoyang Hospital; and Beijing Friendship 
Hospital. All three hospitals are affiliated to the Capital Medical University. The Beijing Obstetrics and Gynecology Hospital is a top maternal and child health care hospital with 660 beds and the number of births exceeding 14,000 every year. The Beijing Chaoyang Hospital is an advanced general hospital, and has a well-known emergency medicine clinical research center. The Beijing Friendship Hospital is also an advanced general hospital, and has a national digestive system disease clinical research center. Patients with APIP generally prefer to go to obstetrics, emergency department, and gastroenterology; therefore, we selected these three hospitals for conducting this research. The aim of the present study was to describe the etiology, clinical manifestations, and maternofetal outcomes of APIP.

\section{Materials And Methods}

Patients

All three hospitals agreed to participate in this study. We reviewed all pregnant women who attended the Chaoyang Hospital between 2015 and 2020, and Beijing Obstetrics and Gynecology Hospital and Beijing Friendship Hospital between 2010 and 2020. The inclusion criterion was acute pancreatitis diagnosed during pregnancy. Patients who developed acute pancreatitis in their puerperium period or patients with chronic pancreatitis were excluded. The study was approved by the institutional review board of the Beijing Obstetrics and Gynecology Hospital. The records and data did not include potential patient identifying information, so informed consent was not required.

APIP diagnosis and definition

The diagnosis and severity categories of APIP were according to the Atlanta Criteria and Clinical practice guideline ${ }^{[8,9]}$. APIP is also classified according to different pathogenic causes: acute gallstone pancreatitis, hypertriglyceridemic pancreatitis, and idiopathic pancreatitis ${ }^{[10,11,12]}$. Different pregnancy stages are defined according to the gestational age, such as the first trimester (1-12 weeks), second trimester (13-28 weeks), and the third trimester (from 29 weeks to before delivery). Data pertaining to the following variables were collected from the medical records: maternal age, gestational age at the time of presentation and delivery, potential causes of APIP, clinical features and complications, diagnostic tests, clinical management, and maternal and infant outcomes.

\section{Statistical analysis}

All data analyses were performed using IBM SPSS 25.0. Categorical variables are presented as frequency (\%) and between-group differences assessed using the Chi-squared test or the Fisher exact test, as appropriate. Normally distributed continuous variables are presented as mean \pm standard deviation (SD) and between-group differences assessed using one-way analysis of variance. Non-normally distributed continuous variables were analyzed using non-parametrical test. All tests were two-tailed, and $\mathrm{P}$ values $<0.05$ were considered indicative of statistical significance.

\section{Results}

\section{Demographics}

During the study reference period, a total of 32 pregnant women qualified the inclusion criteria. The mean maternal age was $30.6 \pm 3.8$ years (range $20-39$ ). The mean gestational age was $33.1 \pm 5.4$ weeks with majority of the episodes occurring in the third trimester $(90.6 \%, 29 / 32)($ Table 1$)$. Approximately $28.1 \%(9 / 32)$ of the patients were 
multiparous, and $71.8 \%$ (23/32) were nulliparous. Two patients $(6.250 \%)$ experienced recurrences during the same pregnancy. Moreover, 4 patients underwent IVF-ET (in vitro fertilization and embryo transfer) and 3 patients had twin pregnancies.

Table 1

Distribution of episodes of acute pancreatitis by trimester

\begin{tabular}{|lllll|}
\hline $\begin{array}{l}\text { Trimester } \\
\mathbf{n}(\%)\end{array}$ & $\begin{array}{l}\text { Age, years } \\
\text { (mean } \pm \text { SD) }\end{array}$ & $\begin{array}{l}\text { Gravidity } \\
(\text { mean } \pm S D)\end{array}$ & $\begin{array}{l}\text { Parity } \\
(\text { mean } \pm S D)\end{array}$ & $\begin{array}{l}\text { GA, weeks } \\
\text { (mean } \pm S D)\end{array}$ \\
\hline 1st 0 (0) & & & & \\
\hline 2nd 3 (9.375) & $33.0 \pm 3.6$ & $2.67 \pm 0.57$ & $0.67 \pm 0.57$ & $20.33 \pm 6.42$ \\
\hline 3rd 29 (90.625) & $30.3 \pm 3.8$ & $1.97 \pm 1.08$ & $0.24 \pm 0.43$ & $34.48 \pm 3.16$ \\
\hline Total 32 (100) & $30.6 \pm 3.8$ & $2.03 \pm 1.06$ & $0.28 \pm 0.45$ & $33.16 \pm 5.41$ \\
\hline GA: gestational age; SD: standard deviation & & \\
\hline
\end{tabular}

\section{Etiology}

The most common causes of APIP were hypertriglyceridemia $(56.2 \%, 18 / 32)$ and gallstones $(28.1 \%, 9 / 32)$. The other causes of APIP were idiopathic $(12.5 \%, 4 / 32)$ and gallstone complicated with hypertriglyceridemia $(3.1 \%$, 1/32) (Table 2). Among all the patients, 56.2\% (18/32) had mild acute pancreatitis (MAP), including 7 patients with hypertriglyceridemia and 11 patients without hypertriglyceridemia. The observed between-group difference in the incidence of MAP was statistically significant $(P=0.025)$, which suggested that patients without hypertriglyceridemia tended to have milder clinical manifestations. 
Table 2

Etiology and clinical characteristics of acute pancreatitis in pregnancy

\begin{tabular}{|c|c|c|c|c|}
\hline & \multicolumn{3}{|c|}{ Other causes } & \multirow[t]{2}{*}{ HTG-AP } \\
\hline & Gallstone & Idiopathic & Mix & \\
\hline Number & $9(28.125)$ & $4(12.5 \%)$ & $1(3.125)$ & $18(56.25)$ \\
\hline \multicolumn{5}{|l|}{ Severity of APIP n (\%) } \\
\hline MAP & 7 & 4 & 0 & 7 \\
\hline$M S A P+S A P$ & 2 & 0 & 1 & 11 \\
\hline Gestational diabetes mellitus n (\%) & 2 & 1 & 0 & 5 \\
\hline Fatty liver disease n (\%) & 2 & 0 & 0 & 3 \\
\hline Pleural effusion n (\%) & 2 & 0 & 0 & 5 \\
\hline Ascites n (\%) & 2 & 0 & 1 & 7 \\
\hline Pelvic effusion n (\%) & 1 & 0 & 0 & 3 \\
\hline Localized complications n (\%) & 1 & 0 & 0 & 3 \\
\hline Organ dysfunction n (\%) & 2 & 0 & 1 & 11 \\
\hline Intrahepatic cholestasis of pregnancy $\mathrm{n}(\%)$ & 0 & 1 & 1 & 0 \\
\hline Timing of diagnosis $\geq 24$ hours $n(\%)$ & 5 & 1 & 0 & 5 \\
\hline Length of stay $\geq 10$ days $n(\%)$ & 5 & 0 & 1 & 12 \\
\hline Amylase level > $172 \mathrm{U} / \mathrm{L}$ & 8 & 1 & 1 & 14 \\
\hline
\end{tabular}

3. Clinical manifestations of APIP

The most frequent presentation of APIP in our cohort was abdominal pain $(93.7 \%, 30 / 32)$. More than half of all patients had nausea and vomiting $(71.8 \%, 23 / 32)$ while fever was less common $(18.7 \%, 6 / 32)$. We compared the commonly used laboratory indices according to the severity of APIP [MAP, moderately severe acute pancreatitis (MSAP), and severe acute pancreatitis (SAP)] (Table 3). Only the level of triglycerides showed a positive correlation with the severity of APIP $(P=0.039)$. However, serum amylase, serum glucose, serum calcium, leukocyte count, total cholesterol, low-density lipoprotein cholesterol, and high-density lipoprotein cholesterol showed no correlation with the severity of APIP (Table 3). 
Table 3

Severity of APIP and abnormality of serum indices

\begin{tabular}{|lllll|}
\hline & MAP & MSAP & SAP & P value $^{\text {a }}$ \\
\hline Number & 18 & 4 & 10 & \\
\hline Age, years & $30.89 \pm 3.27$ & $30.75 \pm 1.708$ & $30.10 \pm 5.527$ & 0.881 \\
\hline Amylase level $>172 \mathrm{U} / \mathrm{L}$ & 12 & 3 & 9 & 0.458 \\
\hline White blood cell count & 11 & 3 & 9 & 0.287 \\
\hline NE $>75 \%$ & 14 & 2 & 10 & 0.074 \\
\hline Hyperglycemia $\geq 7.8 \mathrm{mmol} / \mathrm{L}$ & 2 & 0 & 0 & 0.637 \\
\hline Hypocalcemia $<2 \mathrm{mmol} / \mathrm{L}$ & 4 & 0 & 3 & 0.605 \\
\hline Hypertriglyceridemia $\geq 11.3 \mathrm{mmol} / \mathrm{L}$ & 6 & 3 & 8 & $0.039^{*}$ \\
\hline Total cholesterol $\geq 5.65 \mathrm{mmol} / \mathrm{L}$ & 14 & 4 & 8 & 0.838 \\
\hline Low-density lipoprotein cholesterol $>3.3 \mathrm{mmol} / \mathrm{L}$ & 5 & 1 & 6 & 0.236 \\
\hline High-density lipoprotein cholesterol $<1 \mathrm{mmol} / \mathrm{L}$ & 3 & 3 & 3 & 0.073 \\
\hline Timing of diagnosis $\geq 24$ hours & 5 & 2 & 5 & 0.501 \\
\hline Length of stay $\geq 10$ days & 6 & 3 & 9 & $0.006^{*}$ \\
\hline a Fisher test & & & & \\
\hline
\end{tabular}

\section{Maternal and fetal outcomes}

There were no maternal and fetal deaths in our cohort. However, one patient asked to be discharged from the hospital and gave up treatment. Another patient requested induction of labor because she was afraid that the drugs used during the treatment would be harmful to her fetus. In our study, 59.3\% (19/32) of the patients underwent emergency cesarean section owing to the medical condition. Five $(18.519 \%)$ live births were diagnosed with neonatal respiratory distress syndrome (Table 4). Apgar scores at $1 \mathrm{~min}, 5 \mathrm{~min}$, and $10 \mathrm{~min}$ of the neonates were evaluated in 27 cases (Table 5). Asphyxia in the neonates was correlated with the severity of APIP of the mother $(P=0.039)$. However, the length and weight of the neonates showed no correlation with the severity of APIP (Table 5). Fetal malformations were observed in two cases; one was hypospadias, and the other was gastrointestinal malformation with congenital heart disease. 
Table 4

Maternal and fetal outcomes

\begin{tabular}{|c|c|c|c|}
\hline & MAP & MSAP & SAP \\
\hline Number & 18 & 4 & 10 \\
\hline Cesarean birth & 10 & 2 & 9 \\
\hline Vaginal delivery & 2 & 1 & 0 \\
\hline Continued pregnancy & 6 & 0 & 1 \\
\hline Induction of labor ${ }^{a}$ & 0 & 1 & 0 \\
\hline Postpartum hemorrhage & 6 & 0 & 1 \\
\hline Maternal or fetal death & 0 & 0 & 0 \\
\hline
\end{tabular}

Table 5

Apgar scores of neonates

\begin{tabular}{|c|c|c|c|c|}
\hline & MAP & MSAP & SAP & $P$ value \\
\hline \multicolumn{5}{|l|}{ Apgar score } \\
\hline $1 \min ^{a}$ & $9.67 \pm 0.61$ & $9.33 \pm 0.57$ & $8.56 \pm 1.87$ & 0.298 \\
\hline $5 \min ^{a}$ & $10.00 \pm 0.00$ & $10.00 \pm 0.00$ & $9.22 \pm 1.64$ & $0.039 *$ \\
\hline $10 \min ^{a}$ & $10.00 \pm 0.00$ & $10.00 \pm 0.00$ & $9.22 \pm 1.64$ & $0.039 *$ \\
\hline Length $^{\mathrm{b}}, \mathrm{cm}$ & $45.80 \pm 4.03$ & $48.67 \pm 1.15$ & $45.78 \pm 3.83$ & 0.479 \\
\hline Weight $^{\mathrm{b}}, \mathrm{g}$ & $2478.67 \pm 719.23$ & $2883.33 \pm 261.59$ & $2710.56 \pm 761.42$ & 0.573 \\
\hline \multicolumn{5}{|c|}{${ }^{a}$ non-parametrical test (Kruskal-Wallis H) } \\
\hline bone-way ar & is of variance & & & \\
\hline
\end{tabular}

\section{Discussion}

The present study described 32 cases of APIP with the aim to characterize the clinical correlates of this disease in China. In our cohort, most of the events $(90.6 \%, 29 / 32)$ presented in the third trimester, which suggests an increased incidence with increasing gestational age. Gallstone is the most commonly reported etiology of AP among pregnant women in Europe and America, followed by idiopathic, alcohol abuse, and hypertriglyceridemiainduced $A \mathrm{P}^{[1,2,13-15]}$. In China, alcohol abuse is uncommon during pregnancy because of the excessive concern for pregnant women. However, most pregnant women in China tend to have high-fat diet due to local culture. Besides, 
the plasma lipid levels are liable to increase during pregnancy due to the effects of estrogen, progesterone, and human placental lactogen ${ }^{[16]}$. Lipid levels in the first trimester are usually the same as the pre-pregnancy levels and significant changes in lipid metabolism are usually seen in the second and third trimesters ${ }^{[17]}$. There is usually a two to four-fold rise in plasma triglyceride level during pregnancy. However, this is usually well-tolerated and does not affect the mother or fetus, but in some high-risk women, triglyceride levels may increase to abnormally high levels (more than 95th centile for the age) and in some cases up to severe level (>1000 mg/dL or $11.3 \mathrm{mmol} / \mathrm{L})^{[18]}$. As expected, the most common cause of APIP in Chinese women in this study was hypertriglyceridemia (56.25\%, 18/32). The results are in line with previous studies conducted in China ${ }^{[6]}$. However, compared with the previously reported data, the proportion of APIP cases caused by hyperlipidemia was higher in our study ${ }^{[6,7,19]}$. We may speculate that the rising trend of obesity worldwide may have had a negative impact on the incidence of this disease ${ }^{[6]}$. Many cases of APIP caused by hypertriglyceridemia were also reported in Japan and Korea ${ }^{[20-22]}$. Therefore, the etiological pattern of APIP appears to differ significantly between Asian and European women. Several studies have shown that patients with APIP caused by hypertriglyceridemia have an increased tendency to develop SAP and other complications ${ }^{[1,7,19,23,24]}$. We also observed a similar tendency in our study.

In our study, the initial diagnosis of 10 (31.2\%) patients was different from the discharge diagnosis, and a delayed diagnosis of APIP was made for 12 (37.5\%) patients. Several factors can explain this phenomenon. First of all, enlargement of the uterus displaces the pancreas posteriorly, and some APIP patients may not have the typical clinical manifestation of upper abdominal pain. The clinical manifestations of nausea and vomiting are similar to various physiological or pathological manifestations during pregnancy. Moreover, inflammation in the pancreas can induce uterine contraction; thus, the abdominal discomfort is liable to be mistaken for abdominal discomfort related to labor. Second, pregnancy will affect the changes and interpretation of various blood and biochemical indices. In our study, 25\% (8/32) of patients had an increase in serum amylase less than three times the normal value, and the increase in serum amylase was not related to the severity of APIP. Studies have shown that compared with serum amylase, serum lipase has a higher sensitivity and a larger diagnostic window ${ }^{[25,26]}$. In case of hyperlipidemic acute pancreatitis, lipase offers a better diagnostic accuracy (91.8\%) than amylase $(40.3 \%)^{[27]}$. Moreover, lipase may have an advantage over amylase in determining the severity of acute pancreatitis ${ }^{[25]}$. Regrettably, serum lipase levels were not tested in most of the patients in this study. Thus, based on previous studies, we strongly recommend that serum lipase should be added to the routine biochemical examination of patients with abdominal pain during pregnancy to facilitate the diagnosis of APIP. Third, although ultrasound is still the first abdominal imaging method for APIP patients, the findings are greatly affected by gastrointestinal gas and the posture of pregnant women. Thus, use of ultrasound alone may not be adequate for accurate assessment of APIP. Magnetic resonance imaging (MRI) and Magnetic resonance cholangiopancreatography (MRCP) without iv contrast (gadolinium) should be considered in patients with indeterminate US findings ${ }^{[28]}$.

In the present study, we found that the clinical management of APIP has remained almost the same in the past decade. Treatment of APIP requires multidisciplinary collaboration involving specialists from gastroenterology, radiology, obstetrics, general surgery, neonatology, and even intensive care departments to develop personalized treatment plans. Owing to the lack of standardized guidelines for APIP, the treatment is generally conservative and similar to that administered to non-pregnant patients with acute pancreatitis.

In our study, $55.5 \%$ of gallstone-induced APIP patients had a history of gallstones and $27.7 \%$ of hypertriglyceridemia-induced APIP patients had hyperlipidemia during pregnancy (one of which had a history of hyperlipidemia for two years before pregnancy). Therefore, we recommend primary prevention for high-risk patients 
(history of gallstones, hyperlipidemia or hyperlipidemia during pregnancy, and BMI $\geq 28 \mathrm{~kg} / \mathrm{m}^{2}$ ) before and during pregnancy. For pregnant women with a history of gallstones, we recommend abdominal ultrasound examination during the pre-pregnancy counseling. Timely treatment of patients who are found to have gallstones can help prevent APIP. Patients with hyperlipidemia should stop taking lipid-lowering drugs during pregnancy owing to the lack of definitive evidence of their safety during pregnancy ${ }^{[18]}$. However, they should be fully informed of the possible complications and treatment methods during pregnancy. It is recommended to improve their lifestyle including avoidance of excessive weight gain during pregnancy ${ }^{[24]}$.

The management of APIP is complicated by the decision-making regarding the timing and route of termination of pregnancy (induction of labor or cesarean section or vaginal delivery). Pregnancy termination can also be regarded as a key to achieve cure of $A P^{[29]}$. In our study, pregnancy was terminated in $50 \%(9 / 18)$ of MAP patients and all MSAP and SAP patients $(92.8 \%, 13 / 14)$, except for one patient who became ill at 23 weeks gestation. Based on the treatment of 32 patients, we have summarized some recommendations for termination of pregnancy: (1) Women who agree to the use of fetotoxic medication for pancreatitis treatment or voluntarily terminate their pregnancy; (2) stillbirth, fetal malformations, and severe fetal distress; (3) patients who are in the third trimester and whose condition deteriorates after 24-48 hours of treatment; (4) MSAP and SAP patients. If conditions permit, vaginal delivery should be preferred as it can help avoid infections associated with cesarean delivery. However, for APIP patients whose condition is still worsening after 24 to 48 hours of active treatment (e.g., no improvement in paralytic intestinal obstruction), cesarean delivery should be undertaken immediately to ensure maternal and fetal safety ${ }^{[24]}$.

No maternal or fetal deaths occurred in our study. This observation is in agreement with previous studies ${ }^{[2,6]}$. The good outcomes in our cohort are likely attributable to the improvement in maternal and neonatal intensive and supportive care that have occurred during the past decade in China.

Like all retrospective studies, this study has some limitations. First, because of de-identification of all personal information of patients, follow-up data of APIP patients after their discharge from the hospital were not available. Second, some laboratory indices (such as serum lipase level) were not assessed for all patients. Third, due to the low incidence of APIP, there were only 32 cases in our study. However, the study sample was drawn from a large dataset of pregnant women and so might add valuable practical information to the global knowledge of APIP.

\section{Conclusion}

Acute pancreatitis in pregnancy (APIP) is a rare and serious complication during pregnancy. APIP occurred most commonly in the third trimester, and the main etiology of APIP in our cohort was hypertriglyceridemia.

Hypertriglyceridemia-induced APIP tended to be associated with severe clinical manifestations. Elevated level of triglycerides may serve as a marker of APIP severity. The severity of APIP correlated with higher risk for neonate asphyxia. Appropriate timing of termination of pregnancy in APIP patients is a key imperative. Primary prevention for high-risk patients (history of gallstones, hyperlipidemia or hyperlipidemia during pregnancy, and BMI $\geq 28$ $\mathrm{kg} / \mathrm{m}^{2}$ ) before and during pregnancy is also important. More studies are warranted to further elucidate the etiology, risk factors, and treatment of APIP.

\section{Declarations}

\section{Ethics approval and consent to participate}


I confirm that all methods were performed in accordance with the relevant guidelines and regulations by including a statement in the Ethics approval and consent to participate section to this effect.

The study was approved by the institutional review board of the Beijing Obstetrics and Gynecology Hospital. The records and data did not include potential patient identifying information, so informed consent was not required.

the Beijing Obstetrics and Gynecology Hospital has approved the waiver for need for informed consent.

\section{Consent for publication}

Consent for publication is Not applicable..

\section{Availability of data and material}

\section{The data is availability.}

The datasets generated and/or analysed during the current study are not publicly available due Some reasons but are available from the corresponding author on reasonable request.

The data that support the findings of this study are available from Beijing Obstetrics and Gynecology Hospital but restrictions apply to the availability of these data, which were used under license for the current study, and so are not publicly available. Data are however available from the authors upon reasonable request and with permission of Beijing Obstetrics and Gynecology Hospital.

\section{Competing interests}

The authors declare that the research was conducted in the absence of any commercial or financial relationships that could be construed as a potential conflict of interest.

\section{Funding}

This work was supported by The Beijing Municipal Administration of Hospitals Incubating Program (PX2018078).

\section{Authors' contributions}

Tingting Zhang wrote the main manuscript text. All authors reviewed the manuscript.

\section{Acknowledgements}

This manuscript has been edited and proofread by Medjaden Inc.

The data that support the findings of this study are available from Beijing Obstetrics and Gynecology Hospital but restrictions apply to the availability of these data, which were used under license for the current study, and so are not publicly available. Data are however available from the authors upon reasonable request and with permission of Beijing Obstetrics and Gynecology Hospital.

\section{References}


1. Eddy J J,Gideonsen M D,Song J Y et al. Pancreatitis in pregnancy.[J] .Obstet Gynecol, 2008;112(5): 1075-1081.

2. Ducarme G,Maire F,Chatel P et al. Acute pancreatitis during pregnancy: a review.[J] .J Perinatol, 2014; 34(2): 87-94.

3. Bahiyah A, Thanikasalam KP, Lim HC, Ray JR. Severe acute pancreatitis in pregnancy. Case Rep Obstet Gynecol. 2015;2015:239068.

4. Tang SJ, Rodriguez-Frias E, Singh S et al. Acute pancreatitis during pregnancy. Clin Gastroenterol Hepatol. 2010,8(1):85-90.

5. Vilallonga R, Calero-Lillo A, Charco R, Balsells J. Acute pancreatitis during pregnancy, 7-year experience of a tertiary referral center. Cir Esp. 2014,92(7):468-71.

6. Tang M, Xu JM, Song SS, et al. What may cause fetus loss from acute pancreatitis in pregnancy: Analysis of 54 cases[J]. Medicine (Baltimore), 2018,97(7):e9755

7. Xu Q, Wang S, Zhang Z. A 23-year, single-center, retrospective analysis of 36 cases of acute pancreatitis in pregnancy[J]. Int J Gynaecol Obstet, 2015,130(2):123-126.

8. Banks PA BTL, Dervenis C ea. Classification of acute pancreatitis - 2012: Revision of the Atlanta classification and definitions by international consensus[J]. Gut, 2013,62(1):102-111.

9. Greenberg JA, Hsu J, Bawazeer M et al. Clinical practice guideline: management of acute pancreatitis. Can J Surg. 2016,59(2):128-140.

10. IAP/APA (2013) IAP/APA evidence-based guidelines for the management of acute pancreatitis. Pancreatology 13(4 Suppl 2):e1-15.

11.

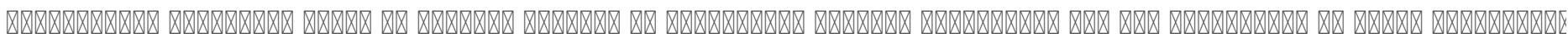

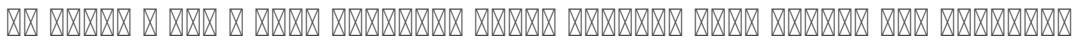

12. Leppäniemi A, Tolonen M, Tarasconi A et al. 2019 WSES guidelines for the management of severe acute pancreatitis. World J Emerg Surg. 2019,13;14:27.

13. Pitchumoni CS, Yegneswaran B. Acute pancreatitis in pregnancy. World J Gastroenterol. 2009,7;15(45):5641-6.

14. Mali P. Pancreatitis in pregnancy: etiology, diagnosis, treatment, and outcomes. Hepatobiliary Pancreat Dis Int. 2016,15(4):434-8.

15. Hot S, Eğin S, Gökçek B et al. Acute biliary pancreatitis during pregnancy and in the post-delivery period. Ulus Travma Acil Cerrahi Derg. 2019,25(3):253-258.

16. Rauschert Sebastian,Gázquez Antonio,Uhl Olaf et al. Phospholipids in lipoproteins: compositional differences across VLDL, LDL, and HDL in pregnant women.[J] .Lipids Health Dis, 2019, 18: 20.

17. Cain Mary Ashley,Ellis Jeremy,Vengrove Marc A et al. Gallstone and Severe Hypertriglyceride-Induced Pancreatitis in Pregnancy.[J] .Obstet Gynecol Surv, 2015,70(9):577-583.

18. Athar S, Ramawat J, Aziz MA et al. Hypertriglyceridemia induced acute pancreatitis in pregnancy: Learning experiences and challenges of a Case report. Clin J Obstet Gynecol. 2019; 2: 006-012.

19. Luo L, Zen $\mathrm{H}, \mathrm{Xu} \mathrm{H}$, et al. Clinical characteristics of acute pancreatitis in pregnancy: experience based on 121 cases[J]. Arch Gynecol Obstet, 2018;297(2):333-339.

20. Sato S, Ohkuchi A, Kawano M et al. Effect of eicosapentaenoic acid agent on aggravated hypertriglyceridemia during pregnancy. J Obstet Gynaecol Res. 2013,39(11):1541-4. 
21. Nishijima K, Takahashi J, Yamamoto M et al. Images in emergency medicine. Pregnant woman with gastric pain. Hypertriglyceridemia-induced pancreatitis. Ann Emerg Med. 2010,55(5):483, 490.

22. Jeon HR, Kim SY, Cho YJ, et al. Hypertriglyceridemia-induced acute pancreatitis in pregnancy causing maternal death. Obstet Gynecol Sci 2016;59:148-151.

23. Sun L , Weiqin L, Geng Y , et al. Acute pancreatitis in pregnancy[J]. Acta Obstetricia et Gynecologica Scandinavica, 2011;90(6):671-676.

24. Cruciat Gheorghe,Nemeti Georgiana,Goidescu Iulian et al. Hypertriglyceridemia triggered acute pancreatitis in pregnancy - diagnostic approach, management and follow-up care.[J] .Lipids Health Dis, 2020, 4;19(1):2.

25. Ismail OZ, Bhayana V. Lipase or amylase for the diagnosis of acute pancreatitis? Clin Biochem. 2017,50(18):1275-1280.

26. Farzaneh F, Esmaeilzadeh A, Rezaei R. Acute Pancreatitis in a Pregnant Women at 30 - 31 Weeks of Gestational Age with Complete Cure. Zahedan J Res Med Sci. 2019,21(3):e91408.

27. Yin G, Cang X, Yu G et al. Different Clinical Presentations of Hyperlipidemic Acute Pancreatitis: A Retrospective Study. Pancreas. 2015,44(7):1105-10.

28. Masselli G., Derme M., Gualdi G. (2018) Acute Abdominal Pain in Pregnant Patients. In: Patlas M., Katz D., Scaglione M. (eds) MDCT and MR Imaging of Acute Abdomen. Springer, Cham. https://doi.org/10.1007/978-3319-70778-5_10

29. Huang C,Liu J,Lu Y et al. Clinical features and treatment of hypertriglyceridemia-induced acute pancreatitis during pregnancy: A retrospective study.[J] .J Clin Apher, 2016, 31(6): 571-578. 